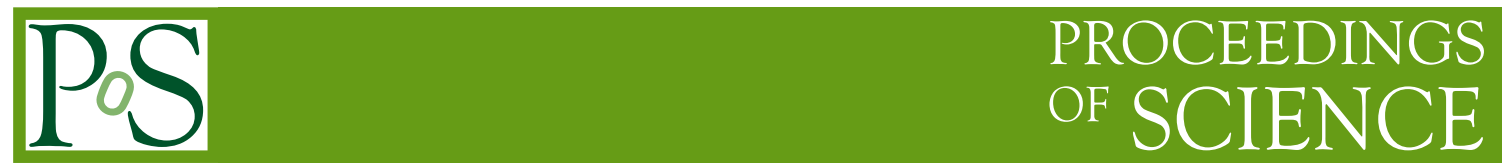

\title{
Heavy flavour phenomenology from lattice QCD
}

\author{
Elvira Gámiz* \\ Fermi National Accelerator Laboratory, Batavia, IL, USA \\ E-mail: egamiz@fnal.gov
}

\begin{abstract}
Heavy quark quantities are useful for testing lattice techniques against well known experimental results, as well as for testing the Standard Model (SM), and searching for physics beyond the SM. I review the results of recent lattice calculations relevant for this program including those of B and $\mathrm{D}$ decay constants and semileptonic decay form factors, and neutral $B$ mixing. The impact of future improvements of lattice results on the clarification of the origin of several disagreements between theory and experiment which are starting to show up are briefly discussed.
\end{abstract}

35th International Conference of High Energy Physics - ICHEP2010,

July 22-28, 2010

Paris France

${ }^{*}$ Speaker. 


\section{Introduction}

Heavy flavour physics plays a dominant role in testing the Standard Model (SM) and searching for New Physics (NP) effects, giving information which is complementary to direct searches in colliders. Some tensions between experimental measurements and theoretical predictions have started to show up in several flavour observables like $\sin (2 \beta)$ or the like-sign dimuon charge asymmetry, and in Unitarity Triangle (UT) analyses. Understanding the origin of these discrepancies is crucial for the flavour program. In order to test the CKM description of the experimentally measured $\mathrm{CP}$-violating and flavour changing processes, we need a precise determination of the weak matrix elements involved in those processes. Lattice QCD can provide those theoretical inputs from first principles and with errors at a few percent level. Accuracy in lattice calculations requires control over all the sources of systematic errors. In particular, it is essential to take into account vacuum polarization effects in a realistic way. In these proceedings I summarize results from new lattice QCD calculations relevant for the heavy flavour program that include effects of at least up and down sea quarks $\left(N_{f}=2,2+1,2+1+1\right)$. In all the calculations quoted in here the main systematic sources of error have been analyzed except for the effects associated to quenching the strange quark.

Simulating heavy quarks on the lattice implies having to deal with discretization errors entering in powers of the mass in lattice units, $a m_{Q}$. These corrections are not negligible at typical lattice spacings $a$. This is particularly dangerous for bottom, for which typically $a m_{b}>1$, so effective theories like heavy quark effective theory (HQET) used by ETMC or the non-relativistic approach (NRQCD) used by HPQCD are still the most adequate framework to describe $b$ quarks on the lattice. For charm, the best strategy is improving the lattice actions to suppress $\left(a m_{Q}\right)^{n}$ corrections and thus keep those corrections under control. Following this strategy, HPQCD is using the Hisq formulation, especially designed to describe charm physics, and ETMC is using the twisted mass (tm) and the Osterwalder-Seiler (OS) formulations. The Fermilab action used for $c$ and $b$ by the FNAL/MILC collaboration starts with an improved Wilson action, which has the same heavy quark limit as QCD. With the Fermilab interpretation in terms of HQET this action can accurately describe $b$ and $c$ without errors that grow as $\left(a m_{Q}\right)^{n}$.

All the calculations mentioned in here are quoting results obtained after extrapolations to the continuum (at least three lattice spacings) and physical quark masses. For a more detailed review, including more technical discussions and references, see, for example, [1].

\section{Decay constants}

The current status of the disagreement between the lattice calculation and the experimental measurement of the decay constant $f_{D_{s}}$, which has generated a vivid interest since 2007, has been discussed in this conference by M. Della Morte [2]. Here I just list the most recent values of $f_{D_{s}}$ and $f_{D}$ from unquenched lattice calculation in Tab. 1.

A similar comparison can be done for $B$ leptonic decays (which could be a sensitive probe of effects from charged Higgs bosons). In this case the conclusion depends on which value of $\left|V_{u b}\right|$ we use to make the comparison between the average of numbers in Tab. 1 and the experimental average $\mathscr{B}\left(B^{-} \rightarrow \tau^{-} \bar{v}\right)=\left(1.72_{-0.42}^{+0.43}\right)$ (BaBar and Belle) [3]. Using the average of exclusive and inclusive 


\begin{tabular}{cccccc}
\hline \hline Collaboration & $f_{D}(\mathrm{MeV})$ & $f_{D_{s}}(\mathrm{MeV})$ & $f_{B}(\mathrm{MeV})$ & $f_{B_{s}}(\mathrm{MeV})$ & $f_{B_{s}} / f_{B}$ \\
\hline HPQCD $\left(N_{f}=2+1\right)$ & $206.3(4.3)$ & $248.0(2.5)$ & $197(13)^{* * *}$ & $240(16)^{* * *}$ & $1.226(26)$ \\
FNAL/MILC $^{*}\left(N_{f}=2+1\right)$ & $220(9)$ & $261(9)$ & $212(8)$ & $256(8)$ & $1.21(2)$ \\
ETMC $\left(N_{f}=2\right)$ & $197(9)$ & $244(8)$ & $194(16)$ & $235(12)$ & - \\
ETMC $\left(N_{f}=2+1+1\right)^{* *}$ & $204(3)$ & $250(3)$ & - & - & - \\
\hline \hline
\end{tabular}

Table 1: Values of $D$ and $B$ meson decay constants from lattice QCD calculations. * labels preliminary results (Lattice 2010) and ${ }^{* *}$ preliminary results (Lattice 2010) with only statist. errors. ${ }^{* * *}$ means published results but with the lattice scale $r_{1}$ changed to the new value calculated by the HPQCD collaboration.

decays $\left(\left|V_{u b}\right|=(3.97 \pm 0.55) \times 10^{-3}\right)$ there is a reasonable agreement $(1.3 \sigma)$ between lattice and experiment, while taken the exclusive value leads to $\mathrm{a} \sim 2.3 \sigma$ disagreement ${ }^{1}$. UT fits are very sensitive to the value of $f_{B}$ and different processes with potential to show up NP effects depend on $f_{B}$ or $f_{B_{s}}$, so any improvement in the decay constants calculations as well as in the understanding of the $\left|V_{u b}\right|^{\text {exc. }} /\left|V_{u b}\right|^{\text {inc. }}$ disagreement is very important.

\section{Semileptonic decays: extraction of CKM matrix elements}

The semileptonic modes $D \rightarrow K l v$ and $D \rightarrow \pi l v$ are very useful not only to extract the values of the CKM matrix elements $\left|V_{c s(d)}\right|$, but to test lattice QCD techniques and methodology by comparing the shape of the corresponding form factors $f_{+}^{D K(\pi)}\left(q^{2}\right)$ to experimental data. There has been a new calculation of $f_{+}^{D K}\left(q^{2}=0\right)$ this year by the HPQCD collaboration [4] which considerably reduces previous errors, $f_{+}^{D K}(0)=0.747(19) \rightarrow\left|V_{c s}\right|=0.961(11)(24)$, where the first error is experimental and second one is the lattice error. The FNAL/MILC and ETMC collaborations are also working in those decays and some examples of their preliminary results are given in Fig. 1. These results are very encouraging since they show a pretty good agreement with experiment.

Another new result this year is the update of the FNAL/MILC calculation of the form factor $h_{A_{1}}(1)$ needed for the extraction of $\left|V_{c b}\right|$ from experimental data on $B \rightarrow D^{*} l v$ [5]. This calculation reduces the theory error to the level of the experimental one, $\left|V_{c b}\right|=39.7(7)(7) \times 10^{-3}$, mainly thanks to improved statistics and the addition of one (smaller) lattice spacing. Again, $\left|V_{c b}\right|$ is a
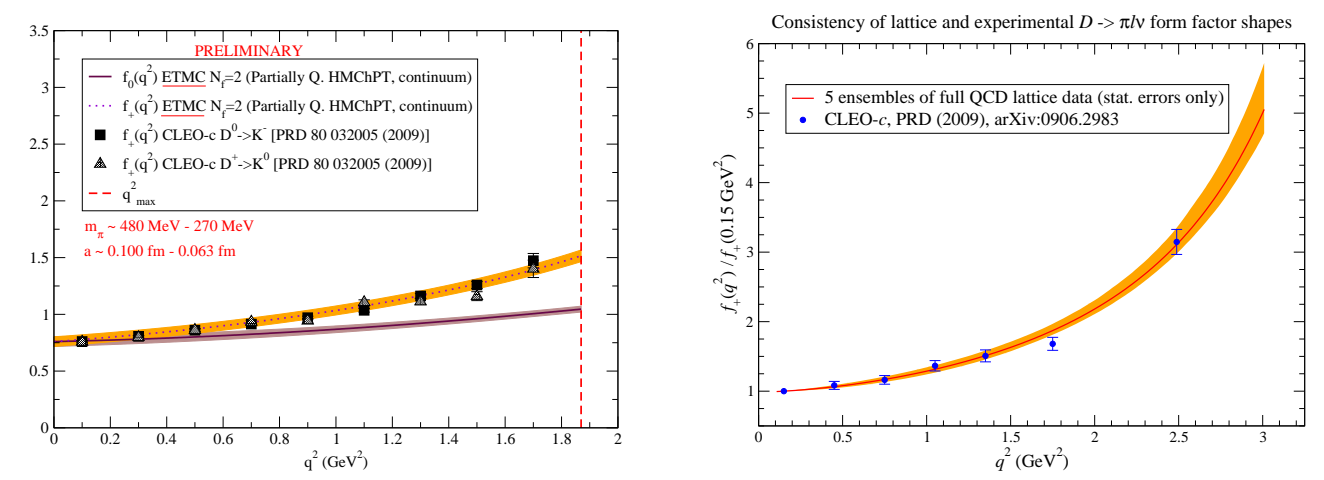

Figure 1: Comparison of experimental and lattice data for $D$ semileptonic decays form factors from the ETMC (plot courtesy of S. Di Vita) and FNAL/MILC collaborations.

\footnotetext{
${ }^{1}$ New results from Belle for $B \rightarrow \pi l v$ seems to point out to an exclusive value of $\left|V_{u b}\right|$ closer to the inclusive one.
} 


\begin{tabular}{ccccc}
\hline \hline collaboration & $f_{B_{s}} \sqrt{\hat{B}_{B_{s}}}(\mathrm{MeV})$ & $f_{B_{d}} \sqrt{\hat{B}_{B_{d}}}(\mathrm{MeV})$ & $\xi$ & status \\
\hline HPQCD & $276(6)(18)$ & $224(9)(12)$ & $1.258(33)$ & final \\
FNAL/MILC & underway & underway & $1.21(5)$ & preliminary \\
RBC/UKQCD & - & - & $1.13(12)$ & exploratory \\
\hline \hline
\end{tabular}

Table 2: $B-$ meson mixing parameters. $\xi$ is defined as the ratio of the parameters in columns 2 and 3 . In the case where there are two errors, the first one is statistical and the second one systematic.

crucial ingredient for UT analyses, so it would be very reinforcing having more lattice calculations of this CKM matrix element, in particular if we take into account the fact that in this case there is also a discrepancy with inclusive determinations.

\section{Neutral $B-$ meson mixing}

The theory-experiment disagreements mentioned before are motivating an increasing interest on the possible impact of NP in neutral $B$ mixing. The theoretical errors associated to the calculation of the non-perturbative $B$ mixing parameters, especially the ratio $\xi$, are one of the most important limiting factors in, for example, UT studies. The current status of unquenched lattice calculations of those parameters is summarized in Tab. 2. HPQCD numbers are corrected for their new, more accurate, value of the lattice scale $r_{1}$. In the exploratory study by the RBC/UKQCD in that table heavy quarks are static [6]. But studies using non-static $b$ by that collaboration, that will also produce values for $f_{B_{s(d)}} \sqrt{\hat{B}_{B_{s(d)}}}$ and will sensibly reduce the error, are underway.

In the near future we will have thus several calculations at the percent level with different lattice formulations for both light and heavy quarks.

\section{Conclusions}

The precise calculation of heavy flavour parameters using lattice QCD is having and will have a very important impact in flavour physics and thus in the search for NP. There has recently been important progress on lattice calculations involving charm quarks as discussed here. We need further improvement in the $B$ sector, where the use of effective theories increase systematic errors. Very precise values for leptonic decays and neutral mixing parameters are starting to be available, but studies using different fermion formulations are desirable and will constitute very important consistency checks. Some collaborations (for example, HPQCD using the Hisq action) are also starting to perform relativistic simulations of $b$ quarks.

\section{References}

[1] J. Heitger, PoS LATTICE2010 (2010) 009.

[2] M. Della Morte, PoS ICHEP2010 (2010) 364, arXiv:1011.5974 [hep-lat].

[3] J. L. Rosner and S. Stone, arXiv:1002.1655 [hep-ex].

[4] H. Na, C. T. H. Davies, E. Follana, G. P. Lepage and J. Shigemitsu, arXiv:1008.4562 [hep-lat].

[5] J. Laiho et al., in preparation.

[6] C. Albertus et al., Phys. Rev. D 82 (2010) 014505 [arXiv:1001.2023 [hep-lat]]. 